\title{
A Novel Unscheduled Islanding Detection Method for Microgrid
}

\author{
Hui $\mathrm{Li}^{1}$, Zhaohan $\mathrm{Li}^{1}$, Zhonghan Guo ${ }^{1}$ and Junwei $\mathrm{Lu}^{2}$ \\ ${ }^{1}$ College of Automation Engineering, Shanghai University of Electric Power, Shanghai, 200090, China \\ ${ }^{2}$ School of Engineering Griffith University Brisbane, QId 4111, Australia
}

\begin{abstract}
Microgrid with its intelligent and flexible control characteristics conform to the trend of sustainable development of electricity, and when the microgrid in the unplanned island state, the successful detection of the island is a prerequisite, energy storage inverter as the key equipment in the microgrid system, island protection is one of the necessary functions. In this paper, an improved islanding detection method based on active frequency drift and q-axis reactive power perturbation is proposed. The method has the advantages of faster detection speed and minor influence on power quality, which makes the energy storage inverter with better output power quality when it works on grid-connected state, and can be detected the islanding state quickly from grid-connected mode to islanded mode. Finally, the validity and superiority of the improved island detection method are verified by simulation experiments.
\end{abstract}

\section{Introduction}

With the rapid development of distributed power generation, more and more renewable energy is converted into electricity sentto the grid through the inverter. When the grid-connected distributed power supply in the event of large power grid failure, the inverter is continue to supply the power to local load and run independently is called the island operation[1]. Taking into account the safety and quality of electricity, should quickly detect the island, take the appropriate control means, eliminating the system malfunction and run again.

At present in the islanding detection, according to different detection methods can be divided into passive and active method. Passive method is applicable in most cases and does not need to introduce hardware circuit, does not affect the power quality of the grid side, but it will fail when the microgrid power matches the load power, so it has a lot of detection blind. The active method includes active frequencydrift (AFD), sliding mode frequency offset method, output power perturbation method, etc. which is adding the disturbance signal actively in the inverter controller to achieve island detection, this method has the advantages of detection timely and small blind spots, but due to the introduction of interference will lead to power grid side of the power quality has declined. Therefore, to solve the contradiction between active detection method and passive detection method, one of the ideas is a combination of a variety of detection algorithms.

At present, the improvement of island detection method can be divided into two categories: faster detection and less harmonic content. Furong Liu analyzed the islanding detection blind zone and proposed a parameter optimization method of active frequency drift with linear positive feedback[2], but this method still causes interference to the power grid. Fenghuang Cai analyzed the harmonic problem after using active frequency drift method, and the harmonic content is reduced without affecting the detection efficiency[3], but it enlarges the detection blind zone while eliminating the harmonic wave. Fangrui Liu improve island detection efficiency or reduce the impact on the network respectively by optimizing the disturbance mode, controller and disturbance parameters[4-5], but only consider the detection effect and ignore the disturbance on the system. In this paper, an improved AFD islanding detection method is proposed, which combines the advantages of active frequency drift detection and the $\mathrm{q}$-axis reactive power perturbation (IQF).

\section{Island detection principle}

As shown in Figure.1, the load power is supplied by the energy storage inverter and the grid when the energy storage inverter is connected to the grid. When the grid is disconnected, the energy storage inverter and the load form an island[6].

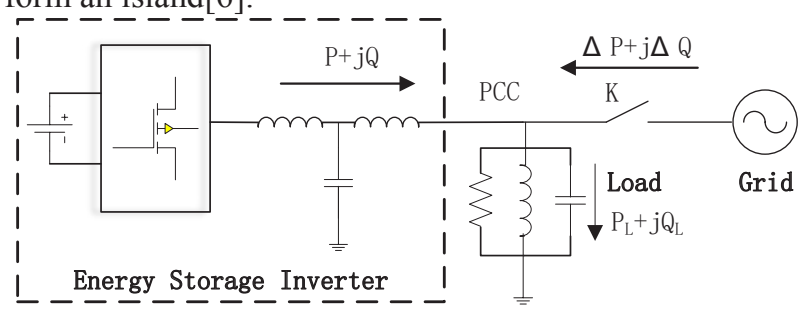

Figure 1.Grid power transmission system for Energy storage inverter. 
The relationship between the branch powers in Figure. 1 can be expressed by equation (1)[7].

$$
\left\{\begin{array}{l}
P_{L}=P+\Delta P \\
Q_{L}=Q+\Delta Q
\end{array}\right.
$$

Where: $\mathrm{P}$ and $\mathrm{Q}$ are the active and reactive power output from the inverter; $\mathrm{P}_{\mathrm{L}}$ and $\mathrm{Q}_{\mathrm{L}}$ are the active and reactive power of the load respectively; and $\mathrm{P}$ and $\mathrm{Q}$ are the active and reactive power output from the grid.

By analyzing Figure 1 get the inverter side voltage, the relationship between the frequency as shown in equation(2)[8].

$$
\left\{\begin{array}{c}
\mathrm{U}=\sqrt{\mathrm{PR}} \\
\mathrm{f}=\mathrm{f}_{0}\left(1-\frac{\mathrm{Q}}{2 \mathrm{PQ}}\right)
\end{array}\right.
$$

Where: $\mathrm{U}$ is the inverter output voltage; $\mathrm{f}$ is the frequency of the inverter side; $Q_{f}=R \sqrt{C / L}$ is the quality factor of the load; $f_{0}=1 / 2 \pi \sqrt{\mathrm{LC}}$ is the resonant frequency of the load; R, L, C are the values of load resistance, inductance and capacitance.

If the power of the inverter does not match the power of the load, the influence of the power variation on the voltage and frequency on the inverter side when the island is generated can be obtained as shown in equations (3) and (4). For example, if the power provided by the inverter is not enough to supply the load $(\Delta \mathrm{P}>0)$, the disconnection of the large power grid will cause the power of the load to decrease $\left(\mathrm{P}_{\mathrm{L}} \downarrow\right)$, According to equation (3) determine the load terminal voltage, power down ( $\mathrm{v} \downarrow$, $\mathrm{f} \downarrow$ ). In such load does not match the conditions, the application of passive island detection method to detect islands.

$$
\begin{array}{r}
\left\{\begin{array}{l}
\Delta \mathrm{P}>0 \rightarrow \mathrm{P}_{\mathrm{L}} \downarrow \rightarrow \mathrm{U} \downarrow \rightarrow \mathrm{f} \downarrow \\
\Delta \mathrm{P}<0 \rightarrow \mathrm{P}_{\mathrm{L}} \uparrow \rightarrow \mathrm{U} \uparrow \rightarrow \mathrm{f} \uparrow
\end{array}\right. \\
\left\{\begin{array}{l}
\Delta \mathrm{Q}>0 \rightarrow \mathrm{Q}_{\mathrm{L}} \downarrow \rightarrow \mathrm{f} \uparrow \\
\Delta \mathrm{Q}<0 \rightarrow \mathrm{Q}_{\mathrm{L}} \uparrow \rightarrow \mathrm{f} \downarrow
\end{array}\right.
\end{array}
$$

If the power of the inverter is matched with the load power, the magnitude and frequency of the voltage and current at the grid-connecting point will not change when the grid is disconnected. At this time, the traditional passive island detection method fails, so the active island detection method needs to be quoted.

\section{Improved island detection technology}

For active islanding detection method, AFD is a more classic and most studied active island detection method, which has the advantages of simple algorithm, easy implementation and fast detection speed. However, because AFD is the frequency of current to add disturbance, and active currency fluctuations will cause the output voltage fluctuations, thus affecting the power quality, so this paper presents an improved AFD method, by adding reactive current disturbance in the $q$ axis, reduce the effect of inverter output power quality.

\subsection{AFD islanding detection method}

As shown in Figure 2, the AFD injects a constant current, slightly varying frequency current into the grid via an inverter. When the grid is disconnected, the frequency of the inverter output voltage is forced to drift until a limit value is exceeded and an isolated island is detected[9].

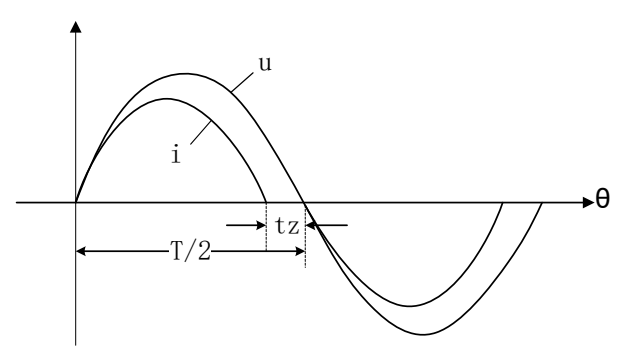

Figure 2. The value of ' $u$ ' and 'i' after the disturbance are added.

Here introduce the concept of the cut-off coefficient $\mathrm{c}_{\mathrm{f}}$, define $c_{\mathrm{f}}$ as the ratio of the time $t_{\mathrm{z}}$ of half-voltage period $\mathrm{T} / 2$ of the zero-crossing of the current zero-crossing lead (or lag) voltage.

$$
\mathrm{c}_{\mathrm{f}}=\frac{2 \mathrm{t}_{\mathrm{z}}}{\mathrm{T}}
$$

According to previous research experience[6], $\mathrm{c}_{\mathrm{f}}$ can be expressed as shown in equation (6).

$$
c_{\mathrm{f}}=\left\{\begin{array}{lr}
\mathrm{c}_{\mathrm{f}} & \text { AFD } \\
\mathrm{c}_{\mathrm{f}}+\mathrm{k}_{\mathrm{f}}\left(\mathrm{f}-\mathrm{f}_{0}\right) & \text { AFDPF }
\end{array}\right.
$$

Where: $c_{f}$ for the initial given truncation coefficient; $k_{f}$ for the feedback gain; $f_{0}$ for the grid rated frequency.

Furong Liu and others have proved through research that the proper selection of the values of $c_{f}$ and $k_{f}$ can speed up the detection of islanding and reduce the detection blind zone. According to the research experience, $\mathrm{c}_{\mathrm{f}}=0.02$ and $\mathrm{k}_{\mathrm{f}}=0.1$ are selected here.

\subsection{The principle of improved island detection method}

Three-phase energy storage inverter control principle shown in Figure 3 . The phase angle $\theta$ of the grid-side phase obtained after the phase-locking is obtained through the perturbation control to obtain the phase angle $\theta^{\prime}$, and the grid-side voltage and current signals are transformed by using the phase angle $\theta^{\prime}$ to obtain grid-side voltage and current values in the synchronous rotating coordinate system $\mathrm{V}_{\mathrm{d}}, \mathrm{V}_{\mathrm{q}}, \mathrm{I}_{\mathrm{d}}, \mathrm{I}_{\mathrm{q}}$; and then $\mathrm{V}_{\mathrm{d}}, \mathrm{V}_{\mathrm{q}}$ and a given active power $\mathrm{P}_{\text {ref }}$, reactive power $\mathrm{Q}_{\text {ref }}$ input power outer ring, thereby calculating the current inner reference $\mathrm{i}_{\text {dref }}, \mathrm{i}_{\text {qref; }}$; obtained by the inner current loop voltage command $\mathrm{u}_{\mathrm{d}}{ }^{*}$, $\mathrm{u}_{\mathrm{q}}{ }^{*}$ After anti-park, anti-clark transform command voltage into the PWM modulation module to generate six PWM signal control energy storage inverter work; finally obtained by the grid side voltage frequency $\mathrm{f}$ to determine whether the island. If it is determined that there is an island, then immediately change the control mode, the power of the outer ring into the voltage outer ring, through a given voltage and frequency reference control energy storage inverter to continue running, and thus from the grid PQ control mode to off-grid VF control mode.

Similar to the single-phase AFD algorithm, the three-phase AFD algorithm detects the output frequency of the energy storage inverter by changing the phase angle 
of the current and achieves the purpose of islanding detection. However, in the three-phase system, the output current is controlled based on the dq rotating coordinate system, and there is a coupling phenomenon between the three phase currents. The phase disturbance also affects the current of the other two phases at the same time, which further deteriorates the waveform. Therefore, this algorithm exists harmonic content of the larger problem.

As shown in Figure 3, unlike the AFD, the new method uses the locked phase angle $\theta^{\prime}$ for the Clark transformation and the park transformation, and uses the frequency f obtained from the phase lock to generate the q-axis interference amount $\mathrm{I}_{\mathrm{qr}}$, Together with the $\mathrm{I}_{\mathrm{q}}, \mathrm{I}_{\text {qref }}$ as the current reference value $\mathrm{I}_{\mathrm{q}}$ of the q-axis.

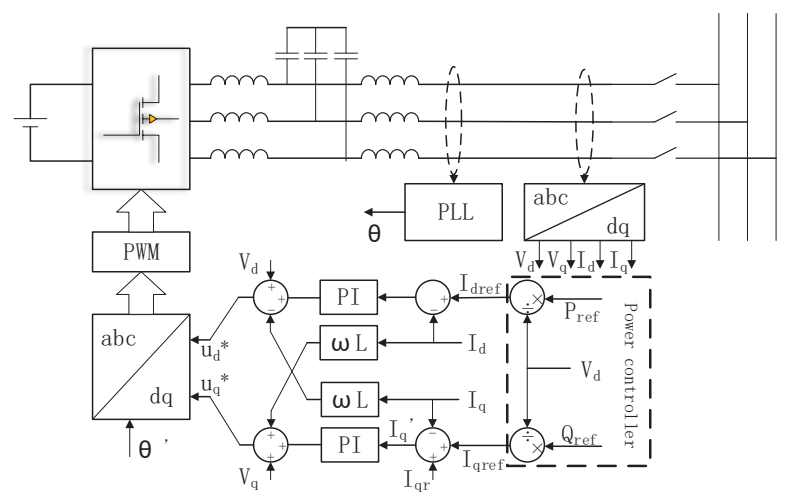

Figure 3. Control diagram of improved AFD island detection.

In [4], the relationship between the q-axis current component $\mathrm{I}_{\mathrm{q}}$ and the grid-side frequency $\mathrm{f}$ is analyzed as shown in equation (7).

$$
I_{q}=-\frac{2 I_{d} Q_{f}}{f_{0}}\left(f-f_{0}\right)
$$

Where: $Q_{f}, f_{0}, I_{d}$ are constants. (7) can be seen as a function of $\mathrm{I}_{\mathrm{q}}$ and $\mathrm{f}$, which can be obtained in Figure 3 after the reactive current $\mathrm{I}_{\mathrm{q}}{ }^{\prime}$ curve equation as shown in equation (8).

$$
I_{q}^{\prime}=-\frac{2 I_{d} Q_{f}}{f_{0}}\left(f-f_{0}\right)+I_{q r}
$$

Where: $I_{\mathrm{qr}}$ is the introduced perturbation.

For $\mathrm{I}_{\mathrm{qr}}$, can be used in different ways of disturbance, the project is more common positive feedback method [4].

$$
I_{q r}=\left[C+K *\left(f-f_{0}\right)\right] * I_{d}
$$

Where: $\mathrm{C}$ is a constant greater than zero; $\mathrm{K}$ is a positive feedback coefficient.

\subsection{Detection strategy of hierarchical perturbation method}

Figure 3 to improve the island detection method by adding $\mathrm{I}_{\mathrm{qr}}$, which avoid the phenomenon of three-phase current coupling, active power affect the voltage and frequency, reactive power only affect the frequency, dq decomposition of the $\mathrm{q}$ axis after the impulse, Will not affect the d-axis active current, that will only affect the frequency, does not affect the voltage, thus ensuring the power quality.

For the island detection of multi-level perturbation algorithm in Figure 3, the application of $\theta^{\prime}$ and $I_{\mathrm{qr}}$ is the key to this paper. The overall control flow chart shown in Figure 4. If the voltage side of the grid frequency exceeds the specified frequency range, determine the island; if not exceeded, to further determine the frequency deviation range, if $0.1 \mathrm{~Hz}$ or less, adding $\mathrm{q}$-axis current interference $\mathrm{I}_{\mathrm{qr}}$, this time for the first disturbance; More than $0.1 \mathrm{~Hz}$, increase the amount of q-axis current interference and the introduction of phase angle interference, until the island is generated, this time for the second disturbance; addition of disturbance, the need to determine the trend of frequency changes, making the direction and frequency of disturbance Change the direction of the same, thus speeding up the detection speed. This algorithm not only guarantees the power quality of the three-phase inverter when the network is connected, but also has a faster island detection speed.

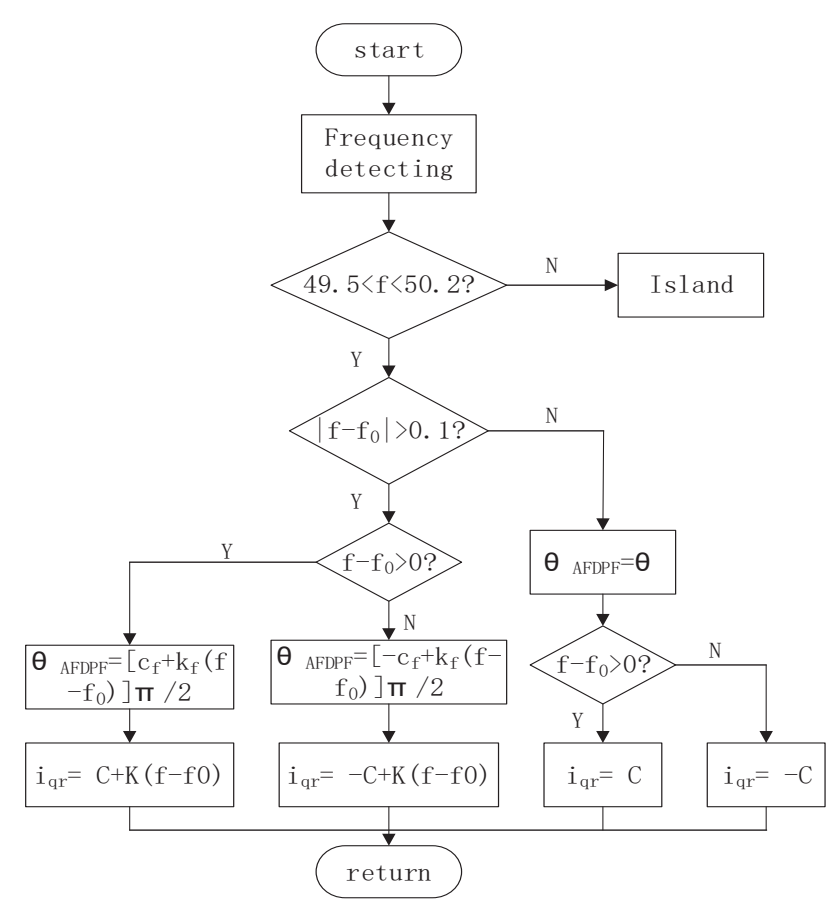

Figure 4.Flow chart of island detection.

\section{Simulation verification and result analysis}

In order to verify the correctness and feasibility of the above theory, simulation experiments are carried out by MATLAB/Simulink.

Simulation, the energy storage inverter began in the grid state, at $\mathrm{t}=0.1 \mathrm{~s}$ disconnect $\mathrm{PCC}$ point. Simulation, will be used passive method, AFD method, IQF method and improved AFD method for the island for island detection. According to GB/T33589-2017 "microgrid access power system technical requirements": the normal frequency of the power system to allow the value of \pm $0.1 \mathrm{~Hz}$, from the country's major power system to run the actual operation, basically maintained at no more than \pm $0.1 \mathrm{~Hz}$ range. And when the common point frequency exceeds $[49.5 \mathrm{~Hz} \sim 50.2 \mathrm{~Hz}]$ range, that the occurrence of the island.

In order to verify the output frequency change of the 
inverter when the island is not matched, verify the correctness of equation (3) in the second section. In this paper, as shown in Figure $1, \Delta \mathrm{P}>0, \Delta \mathrm{P}<0, \Delta \mathrm{Q}>0, \Delta \mathrm{Q}<0$ four conditions. The simulation results are shown in Figure 5.

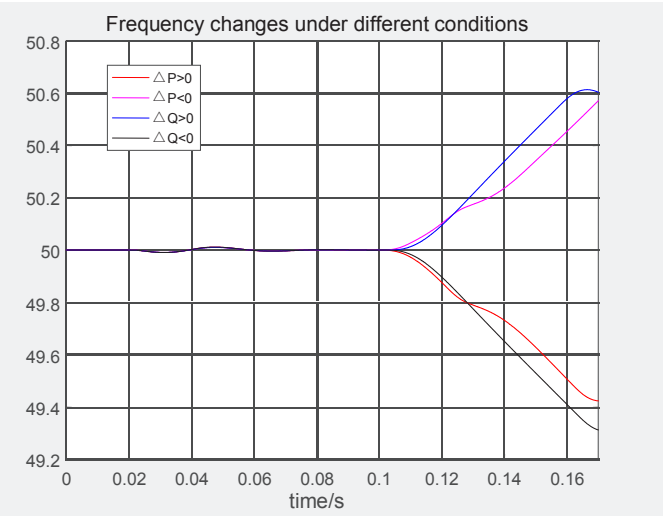

Figure 5.Inverter output frequency under different operating conditions

As can be seen in Figure 5, when $\Delta \mathrm{P}>0, \Delta \mathrm{Q}<0$, an island is formed and the frequency is decreased; when $\Delta \mathrm{P}<0, \Delta \mathrm{Q}>0$, an island is formed and the frequency is increased. Therefore, the experimental results in Figure 5 coincide with those in equation (3), and verify the trend of inverter output frequency during islanding under power mismatch conditions. Island detection provides a theoretical basis. Meanwhile, the voltage is same as the frequency.

In order to verify the effectiveness of the algorithm, this paper simulates the isolated island detection conditions, so that the inverter power and load power match, that is, when using RLC load, then the circuit consumes the capacitive reactive and reactive power cancel each other, The load is equivalent to pure resistive load. The parameters of circuit and simulation shown in Table 1.

Table 1.Simulink parameters.

\begin{tabular}{ll}
\hline Parameter & Value \\
\hline Load(R) / Active & $14.52 \Omega / 10 \mathrm{~kW}$ \\
Load (L) / Reactive & $18.48 \mathrm{mH} / 25 \mathrm{~kW}$ \\
Load $(\mathrm{C}) /$ Reactive & $550 \mathrm{uF} / 25 \mathrm{~kW}$ \\
Load quality factor $\left(\mathrm{Q}_{\mathrm{f}}\right)$ & 2.5 \\
Load resonant frequency $\left(\mathrm{f}_{0}\right)$ & $50 \mathrm{~Hz}$ \\
Rated active power $(\mathrm{P})$ & $10 \mathrm{~kW}$ \\
Rated reactive power $(\mathrm{Q})$ & $0 \mathrm{kvar}$ \\
DC input voltage $\left(\mathrm{U}_{\mathrm{dc}}\right)$ & $800 \mathrm{~V}$ \\
$\mathrm{c}_{\mathrm{f}}$ & 0.02 \\
$\mathrm{k}_{\mathrm{f}}$ & 0.1 \\
$\mathrm{C}$ & 0.02 \\
$\mathrm{~K}$ & 0.1 \\
\hline
\end{tabular}

Set the load resonance frequency $\mathrm{f}_{0}=50 \mathrm{~Hz}$ to match the grid frequency. The test results are shown in Figure 6.

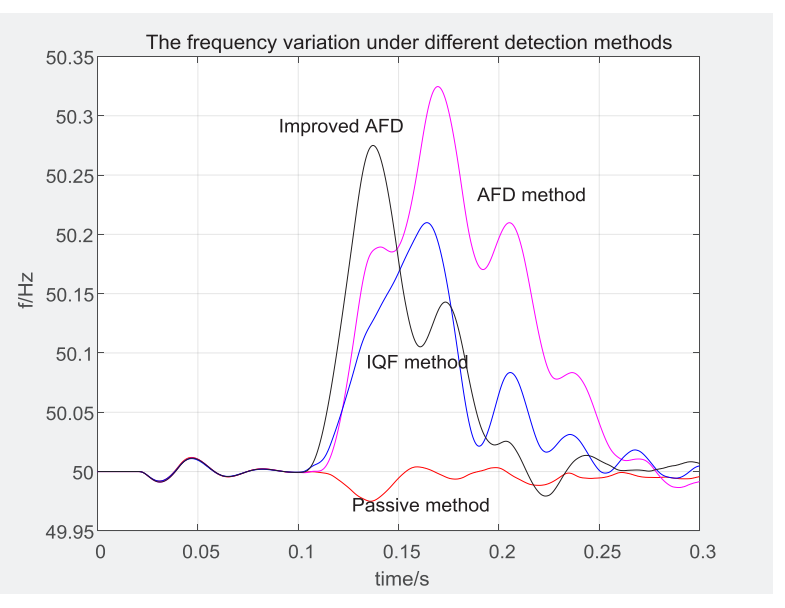

Figure 6.Inverter frequency of different island detection methods.

Before $0.1 \mathrm{~s}$, the energy storage inverter in the grid under the PQ control mode, due to the large grid voltage clamp, the frequency of this time can be stable at $50 \mathrm{~Hz}$. When the $0.1 \mathrm{~s}$ PCC point is broken, the frequency and voltage fluctuate due to the loss of the support of the large power grid, but the change in the frequency detected by the different island detection algorithm is different. Among them, the passive detection method cannot detect the island state; IQF method to detect with about $60 \mathrm{~ms}$; AFD method can be detected within $55 \mathrm{~ms}$ island state; and improved AFD method in $28 \mathrm{~ms}$ to detect the island state. At the same time, the improved AFD method has little effect on the output current of the inverter when the grid is running, and the detection result is shown in Figure 7 Thus confirming the improvement of the fastness of the improved AFD method and the small effect on the power quality.

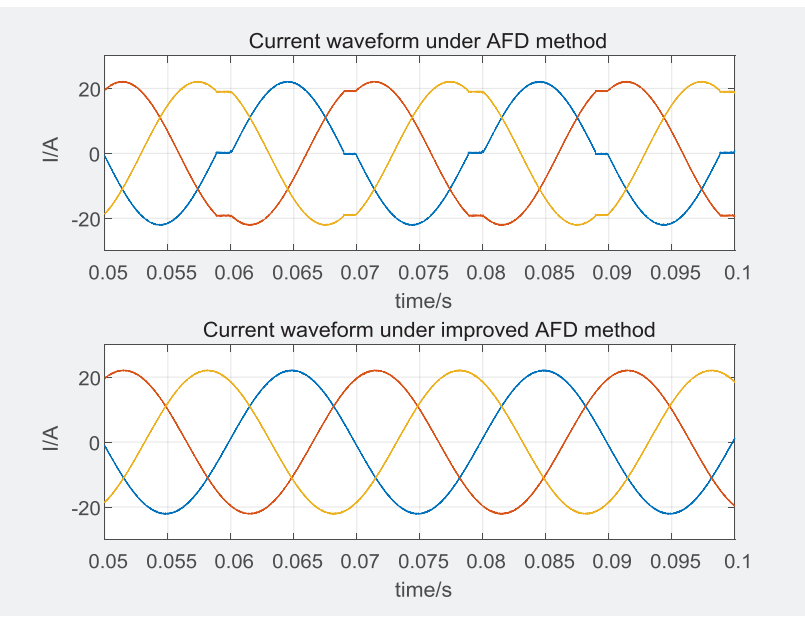

Figure 7.Inverter current of different island detection methods.

\section{Conclusion}

In this paper, an improved islanding detection method based on active frequency drift method and q-axis reactive power perturbation is proposed. The AFD method ensures the speed of detection. The introduction of q-axis 
reactive current disturbance reduces the influence of the output power quality of the inverter.

In this paper, the passive detection method, IQF method, AFD method and the improved AFD method were used to analyze the effect of the method, and the feasibility of the method was verified by simulation experiment. The experimental results show that the improved AFD method has the advantages of faster detection speed and less influence on the output power quality.

Islanding detection is the first step in the study of microgrid and off-grid conversion. How to realize the microgrid security after islanding detection is quickly shifted from the grid-connected mode to the off-grid mode, and how the microgrid bus voltage and frequency in the conversion process is fast stable, that is, to reduce the impact of micro-grid operation mode switching on the power grid and load, is an important issue that needs further study.

\section{Acknowledgment}

This work is supported by Shanghai International Science\&Technology Cooperation Program (No. 15220710500) and Natural Science Foundation of Shanghai (No.15ZR1417600).

\section{References}

1. Guo-Kiang Hung, Chih-Chang Chang and Chern-Lin Chen. Automatic phase shift method for islanding detection of grid connected photovoltaic inverters[J].
IEEE Transaction on Energy Conversion, 2003, 18(1):169-173.

2. LIU Furong, KANG Yong, DUAN Shanxu, et al. Parameter Optimization of Active Frequency - Shift Algorithms [J]. Journal of China Electromechanical Engineering, 2008,28 (1): 95-99.

3. CAI Fenghuang, LIN Qiongbin, WANG Wu. Active Frequency Isolation Detection Method with Harmonic Compensation[J]. Power automation equipment,2014,34(9):54-58.

4. LIU Fangrui, KANG Yong, ZHANG Yu. Parameter Optimization of Active Frequency - Isolated Island Detection with Positive Feedback [J]. New Technology of Electrical Energy, 2008,27 (3): 22-25.

5. SmitaShrivastava, Shailendra Jain, Rajesh Kumar Nema, VijayshriChaurasia. Two level islanding detection method for distributed generators in distribution networks[J]. Electrical Power and Energy Systems, 2017,87: 222-231.

6. ChuttchavalJeraputra, Prasad N. Development of a robust anti-islanding algorithm for utility interconnection of distributed fuel cell powered generation[J]. IEEE Transaction on Power Electronics, 2004, 19(5): 1163-1170.

7. ZHANG Liyan, WU Jun, CHEN Qihong, et al. Small blind fuel cell system and network islanding detection method [J]. Automation of Electric Power Systems,2017,41 (6): 98-102.

8. XIE Qiang. Research on Anti - islanding Detection of Energy Storage Inverter[D].University of Electronic Science and Technology, 2014.

9. CHEN Jiabin, CHEN Alian. An Improved Active Frequency Offset Island Detection Algorithm [J].Journal of Power Supply, 2016. 